\title{
A search model of centralized and decentralized trade
}

\author{
Jianjun Miao \\ 270 Bay State Road, Department of Economics, Boston University, Boston, MA 02215, USA \\ Received 30 April 2004; revised 18 October 2005
}

\begin{abstract}
This paper presents a search model of centralized and decentralized trade. In a centralized market, trades are intermediated by market makers at publicly posted bid-ask prices. In a decentralized market, traders search counterparties. Prices are negotiated and transactions are conducted in private meetings among traders. Traders can choose which market to enter. The determinants of bid-ask spreads and liquidity are analyzed. The welfare consequence of the market fragmentation is also analyzed. It is shown that compared to the competitive market-making, monopolistic market-making may improve social welfare.

(C) 2005 Elsevier Inc. All rights reserved.
\end{abstract}

JEL classification: D4; D83; G12

Keywords: Search; Matching and bargaining; Bid-ask spread; Liquidity; Welfare; Walrasian equilibrium

\section{Introduction}

In the modern economy, some commodities and assets are traded in both centralized and decentralized markets. In centralized markets, trades are intermediated by market makers at publicly posted bid-ask prices. In decentralized markets, traders search counterparties. Prices are negotiated and transactions are conducted in private meetings among traders. For example, in certain securities and futures markets, there are both centralized trading and off-floor trading. In recent years, equity trading is becoming less centralized as the third and fourth market activity has expanded greatly. For example, as documented in Harris (2003, p. 49), for the US equity markets in 2000 , the trading volume in the third market is more than $\$ 2000$ billion $(4.5 \%$ of the

E-mail address: miaoj@bu.edu.

URL: http://people.bu.edu/miaoj. 
overall trading volume) and the trading volume in the fourth market is more than $\$ 7000$ billion ( $16 \%$ of the overall trading volume). ${ }^{1}$

The above observation raises the following questions: What determines liquidity and the bid-ask spread? Why and under what conditions can the centralized and decentralized markets coexist? How does the market fragmentation influence liquidity and the bid-ask spread? What is the social consequence of the market fragmentation?

This paper provides a simple search model to shed light on these questions. The model is based on Rubinstein and Wolinsky (1985) and Gale (1987). At each date, there are potential inflows of new buyers and sellers. Buyers are heterogeneous in their valuation of an asset, while sellers are homogeneous. Buyers and sellers can choose to trade in the centralized market or in the decentralized market. Once a trader makes a transaction, he leaves the economy. Trading in the decentralized market is costly since search incurs time and contact costs. Trading in the centralized market is costly since there are transaction costs for market making.

I analyze and characterize stationary equilibrium. I find that a trader's choice between the two markets has important externalities on other traders. ${ }^{2}$ First, a trader's participation decision depends on other traders' participation decisions. This may cause multiplicity of equilibria. If a trader anticipates that all other traders go to one of the markets, then he will also trade in that market. Thus, concentration of trade may occur. The conditions under which the market fragmentation or concentration can occur depend on the parameter values describing the relative efficiency of the two markets. Second, if a trader enters the centralized market, then he leaves the pool of searchers in the decentralized market. Thus, the bid-ask prices in the centralized market influences the market tightness in the decentralized market, and hence its equilibrium outcome, including negotiated prices and traders' payoffs. These two externality effects are important for understanding the working of the model.

The main results of the paper are as follows. First, I analyze the determinant of the bid-ask spread. ${ }^{3}$ I show that no matter how bid-ask prices are set in the centralized market, there is a positive bid-ask spread as long as the centralized and decentralized markets coexist in equilibrium. Moreover, the average negotiated price in the decentralized market is inside the bid-ask spread (see the supporting experimental evidence reported by Campbell et al., 1991). This result is related to the idea that market makers provide service of immediacy, as pointed out by Demsetz (1968). Thus, the quoted ask price includes a premium for immediate buying and the bid price reflects a concession required for immediate sale.

I also show that the bid-ask spread reflects the transaction cost in the centralized market and the search frictions in the decentralized market. In particular, under competitive market-making, the bid-ask spread is equal to the transaction cost. Under monopolistic market-making, the bidask spread exists even if there is no transaction cost. Further, it is positively related to the search frictions in the decentralized market reflected by the discount rate and contact rate. That is, the bid-ask spread is narrower if traders are more patient or can more easily find other traders. Another testable result is that under both competitive and monopolistic market-making, the bidask spread is positively related to the average negotiated price in the decentralized market.

\footnotetext{
1 The third market includes dealers and brokers who arrange trades in exchange-listed stocks away from an exchange. The fourth market refers to trading in exchange-listed stocks within electronic communications networks.

2 Search externalities are emphasized in the labor market models, e.g., Diamond (1982), Mortensen (1982), and Hosios (1990).

3 The main traditional theories of bid-ask spreads are based on inventory risk (Garman, 1976; Amihud and Mendelson, 1980; and Ho and Stoll, 1981) or asymmetric information (Bagehot, 1971; Glosten and Milgrom, 1985; and Kyle, 1985).
} 
Second, I show that liquidity in the centralized market measured by trading volume is negatively related to the bid-ask spread, and positively related to search frictions in the decentralized market. This result demonstrates that trading volume reflects the relative efficiency of the centralized and decentralized markets because of competition. It is supported by some experimental and empirical studies (e.g., Campbell et al., 1991; Lamoureux and Schnitzlein, 1997; Stoll, 2000).

Third, I establish some limiting results. Specifically, consider perfectly competitive market makers. I show that starting from an equilibrium in which centralized and decentralized markets coexist, the decentralized market is driven out of the economy if the search frictions in the decentralized market becomes large enough, or the transaction cost in the centralized market becomes small enough. A counterpart result is obtained for the centralized market. Furthermore, if either search frictions or transaction costs vanish, then the limiting equilibrium becomes Walrasian in the flow sense of Gale (1987). Similar results are obtained for monopolistic market-making. The only difference is that the decentralized market may not disappear even though the transaction cost converges to zero because there still exists monopoly inefficiency in the centralized market.

Finally, I show that the fragmentation of a centralized market improves social welfare if the bid-ask spread after the fragmentation is small enough. In particular, under competitive market making, the fragmentation always improves social welfare. I also show that the opening of a centralized market in a decentralized market economy may not improve social welfare. These results seem surprising since trading in the centralized market provides immediacy and saves search costs, which should benefit traders. The reasons for my results are as follows: (i) Each transaction in the centralized market incurs a cost. Under the market fragmentation, traders have an additional marketplace to trade, which can save transaction costs. This effect may dominate so that fragmentation improves social welfare. ${ }^{4}$ (ii) The opening of a centralized market in a decentralized market benefit high valuation buyers since low valuation buyers do not enter the centralized market. However, it also imposes negative externalities on the decentralized market since it makes the market tighter. Thus, buyers in the decentralized markets are worse off.

I also study the case where market makers act as a social planner to select bid-ask prices so as to maximize social welfare, given search frictions. Compared to this constrained social optimum, competitive market-making implies too much entry to the centralized market and a too narrow bid-ask spread. By contrast, monopolistic market-making may cause too little entry to the centralized market and a too wide bid-ask spread. Importantly and surprisingly, compared to the competitive market-making, monopolistic market-making may improve social welfare, because it partially internalizes the externalities of bid-ask prices on the decentralized market. This might explain why the monopolistic specialists system on the New York Stock Exchange could be socially useful.

This paper is related to the literature on the search models of exchange. As mentioned earlier, the seminal papers are Rubinstein and Wolinsky (1985) and Gale (1987). Mortensen and Wright (2002) extend these papers by adding pecuniary search costs and considering a general matching technology and bargaining rule. They show that constrained efficiency can be obtained if thirdparty market makers set up a complete set of submarkets and traders can select into appropriate submarkets.

Rubinstein and Wolinsky (1987) introduce middlemen into a search model. Their model has been generalized by a number of papers (e.g., Li, 1998; Shevchenko, 2004; and Masters, 2004).

\footnotetext{
4 See Campbell et al. (1991) for experimental evidence.
} 
In my model, intermediation is conducted by market makers, instead of middlemen. As a result, all traders can trade at publicly posted bid-ask prices. This is different from the middlemen model in which traders can search for and bargain with middlemen or counterparties. My model is related to Gehrig's (1993), who studies a static search model with market makers. However, my dynamic model has many implications different from Gehrig (1993). ${ }^{5}$ Spulber (1996) and Rust and Hall (2003) study dynamic search models of intermediation, but they do not consider traders searching each other in the decentralized market. Neeman and Vulkan (2003a) provide a different model of centralized and decentralized trade. They show that the entry of a market maker causes a complete unraveling of direct negotiations, and in perfect equilibrium almost all trade takes place in the centralized market.

The link between liquidity and search is pointed out by Lippman and McCall (1986). The issue of liquidity, concentration and fragmentation of trade across markets is studied by Pagano (1989) in a static model without intermediaries. Similar questions are analyzed in models based on asymmetric information or inventory risk (e.g. Mendelson, 1987; Biais, 1993; Madhavan, 1995).

My model is closely related to several search models of asset markets initiated by Duffie et al. (2003a). Duffie et al. (2003b) generalize Rubinstein and Wolinsky's (1987) model. Although their paper differs from mine in terms of addressed questions and modeling details, they also show that the bid-ask spread is smaller if traders can find other traders more easily. The underlying mechanism is very different. In their model, increased search efficiency improves a trader's bargaining position relative to the middlemen, while in my model it provides competitive pressure on the centralized market. Vayanos and Wang (2002) generalize Duffie et al. (2003a) and consider that traders can trade two identical assets in two decentralized markets. They study the welfare implication of the concentration of liquidity. Weill (2004) extends Duffie et al. (2003a) to study the implications of search frictions for liquidity when the market makers' inventories "lean against" the outside order flow.

The remainder of the paper is organized as follows. Section 2 presents a search model without intermediaries. Section 3 introduces a centralized market and analyzes equilibria with competitive market makers and with a monopolistic market maker. Section 4 conducts welfare analysis. Section 5 concludes. Proofs are relegated to an appendix.

\section{Trade in the decentralized market}

As a benchmark, I start with the case where there is no intermediary and all trades are conducted in the decentralized market. I model trades in the decentralized market as a process of search, matching and bargaining (see Rubinstein and Wolinsky, 1985; Gale, 1987; Mortensen and Wright, 2002).

Time is continuous and continues forever. At each date, there are $f_{B}$ buyers and $f_{S}<f_{B}$ sellers potentially entering the market. ${ }^{6}$ A trader enters the market if his expected payoff is positive and only if his expected payoff is non-negative. All traders are risk neutral. Each seller has one unit of an asset (or indivisible good) to sell and each buyer demands only one unit of the

\footnotetext{
5 For example, Gehrig's model implies that trading volume is independent of search costs or transaction costs.

6 This assumption will be clear from Eq. (9) below. Note that I assume there are independent flows of buyers and sellers. This greatly simplifies the analysis relative to Duffie et al. (2003a, 2003b), where agents are initially buyers, then hold the asset, and later on become sellers. This simplification comes at a cost because, for some parameter values, there does not exist a steady-state equilibrium.
} 
asset. Once a buyer buys or a seller sells, he exits the market. Buyers are heterogeneous in their valuations. Each buyer's valuation $v$ is drawn from a uniform distribution over $[0,1]$. All sellers are homogeneous and can hold the asset without cost.

I focus on the steady state, in which there are $M_{B}$ buyers and $M_{S}$ sellers in the market at each date. These numbers will be determined endogenously in equilibrium. Moreover, there is a stationary distribution of buyers $F$ in the market. This distribution will also be endogenously determined in equilibrium. It may be different from the exogenous uniform distribution of potential entrants since not everyone enters.

\subsection{Matching and bargaining}

Search frictions are modeled in two dimensions:

(i) There is an implicit time cost in that all traders discount future values by the discount rate $r>0$.

(ii) There is an explicit search cost in that a trader contacts another trader randomly.

Assume that a trader contacts another trader according to a Poisson process with intensity $\rho>0$. Since a trader is a buyer with probability $\gamma=\frac{M_{B}}{M_{S}+M_{B}}$, a seller meets a buyer with rate $\gamma \rho$ and a buyer meets a seller with rate $(1-\gamma) \rho$. Note that in order to derive intuitive closed-form solution, I assume a simple linear matching technology throughout the paper. An analysis for general matching technology is beyond the scope of the present paper.

Once a buyer meets a seller, they negotiate a price to trade. For simplicity, suppose one of the two, chosen at random, announces a take-it-or-leave-it price offer. Let $\theta \in(0,1)$ be the probability the buyer makes the offer. If an offer is rejected, the traders part and continue searching; if the offer is accepted, exchange occurs and they leave the market. ${ }^{7}$

This bargaining protocol is equivalent to the generalized Nash solution. To see this, let $V_{B}(v)$ be the expect payoff of a buyer with valuation $v$ and $V_{S}$ be the expected payoff of a seller. Then the negotiated price

$$
p(v)=\theta V_{S}+(1-\theta)\left(v-V_{B}(v)\right)
$$

is the solution to the following problem:

$$
\max _{p}\left(v-p-V_{B}(v)\right)^{\theta}\left(p-V_{S}\right)^{1-\theta},
$$

subject to

$$
v-p \geqslant V_{B}(v), \quad p \geqslant V_{S} .
$$

The parameter $\theta$ can be interpreted as the relative bargaining power of the buyer.

\subsection{Value functions}

It can be shown that the value function $V_{B}(v)$ satisfies the following Bellman equation:

$$
r V_{B}(v)=\rho(1-\gamma) \max \left\{v-p(v)-V_{B}(v), 0\right\} .
$$

\footnotetext{
7 Note that in this bargaining problem, I assume there is complete information about a buyer's valuation for the asset. This assumption simplifies the analysis because bargaining under incomplete information is a hard problem to deal with in a search-and-matching model.
} 
The interpretation is as follows. At any date, the buyer with valuation $v$ meets a seller with probability $\rho(1-\gamma)$. If he trades with the seller he obtains capital gains $v-p(v)-V_{B}(v)$. Otherwise, he has no capital gains. Thus, Eq. (4) is similar to an asset pricing equation.

Similarly, the seller's value function satisfies the following Bellman equation:

$$
r V_{S}=\rho \gamma E_{F}\left[\max \left\{p(v)-V_{S}, 0\right\}\right],
$$

where $E_{F}$ denotes the expectation operator with respect to the distribution $F$.

Using (1), one can rewrite the above Bellman equation as

$$
\begin{aligned}
& r V_{B}(v)=\rho(1-\gamma) \theta \max \left\{v-V_{B}(v)-V_{S}, 0\right\}, \\
& r V_{S}=\rho \gamma(1-\theta) E_{F}\left[\max \left\{v-V_{B}(v)-V_{S}, 0\right\}\right] .
\end{aligned}
$$

Since it can be verified that $V_{B}(v)$ is increasing in $v$, there exists a cutoff value $R>0$ such that only buyers with valuation $v \geqslant R$ have non-negative gains from trade. The cutoff value $R$ satisfies

$$
R-V_{B}(R)-V_{S}=0, \quad V_{B}(R)=0,
$$

and $V_{B}(v)=0$ for $v<R$. Thus, only buyers with valuation $v \geqslant R$ enter the market. Moreover, every meeting results in trade since $v-V_{B}(v)-V_{S} \geqslant 0$ for $v \geqslant R$.

Equation (8) also determines a seller's value function, $V_{S}=R$. Thus, a seller's expected payoff is equal to the marginal participating buyer's valuation. The intuition is simple. When a seller meets the marginal participating buyer, they trade at the price equal to the marginal valuation. Since sellers are homogeneous, this marginal valuation is the common reservation value of all sellers. Note that at each date all $f_{S}$ sellers will enter the market since $V_{S}=R>0$.

\subsection{Flow market-clearing condition}

In a steady state, the following condition must be satisfied:

$$
f_{B}(1-R)=(1-\gamma) \rho M_{B}=\rho \gamma M_{S}=f_{S} .
$$

The first and last equalities require that the inflow and outflow of traders balance. The second equality says that buyers and sellers exit the market in pairs. Note that $R$ is the Walrasian equilibrium price in the flow sense of Gale (1987) since the flow demand $(1-R) f_{B}$ is equal to the flow supply $f_{S}$.

\subsection{Search equilibrium}

I now define equilibrium. A steady state search equilibrium without intermediaries can be described by the value functions $\left(V_{B}, V_{S}\right)$, the negotiated price $p(v)$, the marginal participating type $R$, the stocks of buyers and sellers in the market $\left(M_{B}, M_{S}\right)$, and the distribution of buyers in the market $F$, such that

(i) $\left(V_{B}, V_{S}\right)$ satisfies (6) and (7),

(ii) $p(v)$ is given by (1),

(iii) $R$ satisfies (8), and

(iv) $\left(M_{B}, M_{S}\right)$ satisfies the flow market-clearing condition (9). 
To solve for an equilibrium, first observe that the stationary distribution of buyers in the market is uniform and its density is given by $\mathrm{d} F(v)=\frac{\mathrm{d} v}{1-R}$. Next, it follows from (9) that $R=1-f_{S} / f_{B}$. Thus, the cutoff value $R$ is determined exclusively by the flows of entrants.

Now, substituting $V_{S}=R$ into (6) and simplifying yield the buyer value function

$$
V_{B}(v)=\frac{\rho \theta(1-\gamma)}{r+\rho \theta(1-\gamma)}(v-R), \quad \text { for } v \geqslant R .
$$

Inserting (10) and $V_{S}=R$ into (1) yields the negotiated price

$$
p(v)=R+\frac{r(1-\theta)}{r+\rho(1-\gamma) \theta}(v-R) .
$$

Substituting $V_{S}=R$ and (10) into (7) and simplifying yield an equation for $\gamma$

$$
R=\frac{\rho \gamma(1-\theta)}{r+\rho(1-\gamma) \theta} \int_{R}^{1}[v-R] \frac{\mathrm{d} v}{1-R} .
$$

Solving this equation yields the equilibrium matching probability $\gamma$,

$$
\gamma=\frac{2 R(r+\rho \theta)}{\rho(2 R \theta+(1-\theta)(1-R))} .
$$

Given the value of $\gamma$, the number of buyers and sellers, $M_{B}$ and $M_{S}$, can be solved from (9), and an equilibrium is constructed.

I now summarize the above discussion in the following proposition.

Proposition 1. If the parameter values $\left(r, \theta, \rho, f_{B}, f_{S}\right)$ satisfy

$$
1-\frac{f_{S}}{f_{B}}=R<\frac{\rho(1-\theta)}{2 r+\rho(1-\theta)},
$$

then there exists a unique search equilibrium without intermediaries.

One can check that condition (14) is equivalent to the requirement that the equilibrium matching probability $\gamma \in(0,1)$. This condition is violated if, for example, the buyer's bargaining power $\theta$ is large enough. When $\theta$ is large, a seller would capture a small fraction of trade surplus. Since a seller's equilibrium expected payoff $V_{S}$ is equal to the constant $R=1-f_{S} / f_{B}$, to satisfy Eq. (7), a seller must meet a buyer with a sufficiently high probability $\gamma$. When $\theta$ is large enough, $\gamma$ exceeds 1 , leading to a contradiction.

It is intuitive that traders can negotiate lower prices, when they are more patient, or when they can contact each other more easily, or when buyers have more bargaining power. This intuition is formalized in the following proposition.

Proposition 2. Let the assumption in Proposition 1 hold. Then the negotiated price $p(v)$ is increasing with $r$, and decreasing with $\rho$ and $\theta$ for all $v>R$.

Since the support of $F,[R, 1]$, does not depend on $r, \rho$, and $\theta$, the above result also holds for the expected negotiated price $E_{F}[p(v)]$.

Finally, the limiting equilibrium becomes Walrasian as the cost of search vanishes. A similar limiting result for $r$ is obtained by Gale (1987).

Proposition 3. If $r \rightarrow 0$, or if $\rho \rightarrow \infty$, then the limiting search equilibrium is Walrasian. 


\section{Search equilibrium with market makers}

I now introduce a centralized market, in which traders can observe bid and ask prices and trades are intermediated by market makers. Traders have the option to trade in the centralized or decentralized market. Market makers remain in the market forever and do not hold inventory. Their role is to channel trade between buyers and sellers. A transaction incurs a fixed cost $k$. For now, I assume that in each period there are constant bid and ask prices $b$ and $a$, publicly posted in the centralized market, and do not study how market makers determine these prices. I will consider this issue by analyzing two cases in Sections 3.4-3.5. In the first case, there is a unit mass of identical perfectly competitive risk-neutral market makers. In the second case, there is a monopolistic risk-neutral market maker.

Before I turn to the formal model, I briefly describe the equilibrium when only the centralized market is available. It is clear that only buyers with valuation $v \geqslant a$ enter the market, and that sellers enter the market if their payoffs are nonnegative. In the steady state, the flow marketclearing condition must be satisfied $(1-a) f_{B}=f_{S}$. Thus, the ask price $a=1-f_{S} / f_{B}=R$. If market makers are competitive, then they make zero profit and the bid price $b=a-k$. If there is a monopolistic market maker, the bid price is set to $b=0$. To ensure that all sellers enter the market in equilibrium, I assume $0 \leqslant k \leqslant 1-f_{S} / f_{B}=R$.

\subsection{Matching}

As in the previous section, I focus on the steady state, in which there are $N_{S}$ sellers and $N_{B}$ buyers in the market at each date. These numbers will be endogenously determined in equilibrium. Since centralized trade is instantaneous and traders leave the market immediately after trade, the stock of traders in the centralized market is equal to zero. Thus, $N_{S}\left(N_{B}\right)$ essentially describes the stock of sellers (buyers) in the decentralized market. ${ }^{8}$ Again, assume that a trader contacts another trader according to a Poisson process with intensity $\rho>0$. Let $\alpha$ denote the probability of a buyer in the decentralized market. Then $\alpha=\frac{N_{B}}{N_{B}+N_{S}}$. Thus, a seller meets a buyer with rate $\rho \alpha$ and a buyer meets a seller with rate $\rho(1-\alpha)$.

\subsection{Value functions and marginal valuation}

At each date, a new entrant faces the following problem. He first chooses which market to enter. If he decides to enter the centralized market and if he is a buyer with valuation $v$, then he buys the asset from the market makers at the ask price $a$ and obtains utility $v-a$. On the other hand, if he is a seller, then he sells the asset to the market makers at the bid price $b$ and obtains utility $b$. After trade, the trader leaves the economy. If the trader chooses to enter the decentralized market, then he has to find a counterparty and negotiates a price. After trade, he leaves the economy. If the trader does not meet a counterparty, then he waits and has to make the same decisions described above again.

Formally, if a buyer with valuation $v$ meets a seller in the decentralized market, they negotiate a price $p(v)$, which is determined as in Section 2.1. That is,

$$
p(v)=\theta U_{S}+(1-\theta)\left(v-U_{B}(v)\right),
$$

where $U_{S}$ and $U_{B}(v)$ are the expected payoffs of a seller and a buyer with valuation $v$.

\footnotetext{
8 I would like to thank an anonymous referee for pointing this out and for suggestion of changes in Section 3.6.
} 
The expected payoffs of a buyer and a seller satisfy the following Bellman equations if they trade in the decentralized market:

$$
\begin{aligned}
& r U_{B}(v)=\rho(1-\alpha) \max \left\{v-p(v)-U_{B}(v), 0\right\}, \\
& r U_{S}=\rho \alpha E_{G}\left[\max \left\{p(v)-U_{S}, 0\right\}\right],
\end{aligned}
$$

where $G$ is the conditional distribution of buyers in the decentralized market and will be determined in equilibrium.

As in the previous section, there is a cutoff value $R$ such that only buyers with valuation $v \geqslant R$ enter the markets. ${ }^{9}$ Moreover, every meeting results in trade. The cutoff value $R$ satisfies

$$
R-U_{B}(R)-U_{S}=0, \quad U_{B}(R)=0,
$$

and $U_{B}(v)=0$ for $v<R$. Thus, $U_{S}=R$. It follows that in order to have both trade in centralized and decentralized markets, the market makers must set the bid price $b=R$ such that sellers are indifferent between the two markets.

Since it can be verified that $v-U_{B}(v)$ given below is increasing in $v$, there is a marginal buyer $v_{m}$ such that buyers with valuation $v \geqslant v_{m}$ go to the centralized market and buyers with valuation $v \in\left[R, v_{m}\right]$ engage in direct trade (see Fig. 1). Substituting (15) and $U_{S}=R$ into (16), one can show that

$$
U_{B}(v)=\frac{\rho \theta(1-\alpha)}{r+\rho \theta(1-\alpha)}(v-R) \text { for } R \leqslant v \leqslant v_{m} .
$$

Note that $U_{B}(v)$ is a linear function of $v$ with a slope less than 1 . The cutoff value $v_{m}$ satisfies

$$
v_{m}-a=U_{B}\left(v_{m}\right) .
$$

That is, the marginal buyer $v_{m}$ is indifferent between trading in the decentralized market and in the centralized market. Fig. 1 illustrates the determination of $v_{m}$.

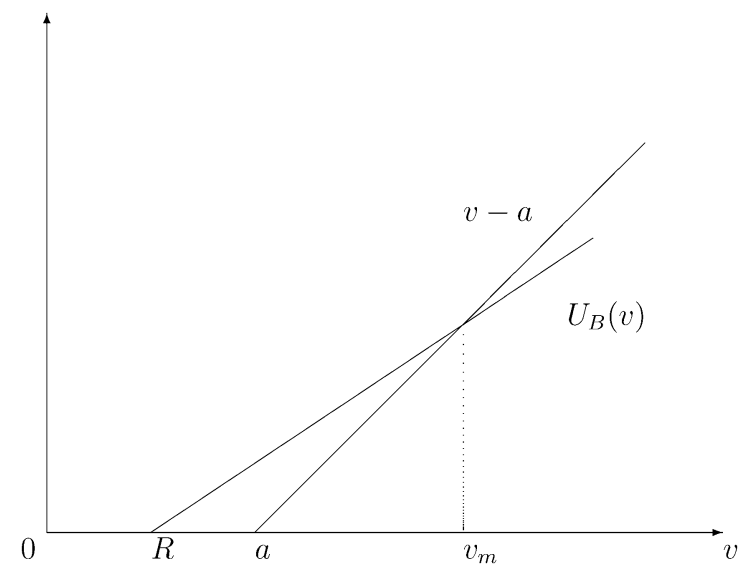

Fig. 1. The determination of the cutoff value $v_{m}$.

\footnotetext{
9 I use the same notation $R$ to denote the cutoff values in two different models described in the previous section and this section, because both cutoff values are equal to the same value $1-f_{S} / f_{B}$, as required by (9), and (21)-(22).
} 


\subsection{Flow market-clearing conditions}

By the analysis in the previous subsection, buyers with valuation $v \in\left[v_{m}, 1\right]$ enter the centralized market and buyers with valuation $v \in\left[R, v_{m}\right]$ trade in the decentralized market. Thus, the flows of buyers entering the centralized and decentralized markets are given by $\left(1-v_{m}\right) f_{B}$ and $\left(v_{m}-R\right) f_{B}$, respectively. Let $\lambda_{S}$ be the fraction of sellers entering the centralized market. Since all $f_{S}$ sellers enter the centralized and decentralized markets, the flows of sellers entering the centralized and decentralized markets are given by $\lambda_{S} f_{S}$ and $\left(1-\lambda_{S}\right) f_{S}$, respectively. To maintain a steady state, the following flow market-clearing conditions must hold:

$$
\begin{aligned}
& \left(1-v_{m}\right) f_{B}=\lambda_{S} f_{S}, \\
& \left(v_{m}-R\right) f_{B}=\rho(1-\alpha) N_{B}=\rho \alpha N_{S}=\left(1-\lambda_{S}\right) f_{S} .
\end{aligned}
$$

Equation (21) describes the condition to clear the centralized market. Equation (22) describes the condition to clear the decentralized market. Its interpretation is similar to that for (9).

\subsection{Equilibrium}

I first study partial equilibria with given bid-ask prices. I defer the analysis of the profit maximizing market-making and the determination of bid and ask prices in later subsections. A (steady-state) search equilibrium with market makers quoting bid-ask prices $(a, b)$ is defined by value functions for buyers and sellers $\left(U_{B}, U_{S}\right)$, the negotiated price $p(v)$ in the decentralized market, the marginal participating types $\left(R, v_{m}\right)$, the stocks of buyers and sellers in the markets $\left(N_{B}, N_{S}\right)$, the distribution $G$ of buyers in the decentralized market, and the fraction of sellers trading in the centralized market $\lambda_{S}$, such that

(i) $\left(U_{B}, U_{S}\right)$ satisfies (16) and (17),

(ii) $p(v)$ satisfies (15),

(iii) $\left(R, v_{m}\right)$ satisfies (18) and (20), and

(iv) $\left(N_{B}, N_{S}, \lambda_{S}\right)$ satisfies the flow market-clearing conditions (21) and (22).

It can be seen that there may exist two degenerate equilibria where traders concentrate on only one market: If all buyers or sellers conjecture that there is no counterparty trading in one of the markets, then there is no trade on that market. ${ }^{10}$ A similar multiplicity of equilibria issue is addressed in Pagano (1989) and Gehrig (1993). Here, I focus on the equilibrium where the two markets coexist, and analyze the degenerate case by studying the limits. The following proposition provides existence and characterization of the unique nondegenerate equilibrium.

Proposition 4. If the ask price $a$ and parameter values $\left(r, \rho, \theta, f_{B}, f_{S}\right)$ satisfy the following conditions:

$$
\begin{aligned}
& \max \left\{\frac{R r}{r+\rho \theta}+\frac{R \theta(2 r+\rho(1-\theta))}{(1-\theta)(r+\rho \theta)}, R+\frac{2 R r}{\rho(1-\theta)}\right\} \\
& <a<\frac{r}{r+\rho \theta}+\frac{R \theta(2 r+\rho(1-\theta))}{(1-\theta)(r+\rho \theta)},
\end{aligned}
$$

$\overline{10}$ Note that market makers do not hold any inventory. 
where $R=1-f_{S} / f_{B}$, then there exists a unique nondegenerate search equilibrium with market makers quoting the bid price $b=R$ and the ask price $a$. In equilibrium, the matching probability is given by

$$
\alpha=\frac{2 R r}{\rho(a-R)(1-\theta)},
$$

the marginal valuation is given by

$$
v_{m}=\frac{a(1-\theta)(r+\rho \theta)-R \theta(2 r+\rho(1-\theta))}{(1-\theta) r},
$$

and the negotiated price in the decentralized is given by

$$
p(v)=R+\frac{r(1-\theta)}{r+\rho(1-\alpha) \theta}(v-R) .
$$

Note that condition (23) is equivalent to the conditions $\alpha \in(0,1)$, and $v_{m} \in(R, 1)$. The latter conditions guarantee that $N_{S}, N_{B}>0$, and $\lambda_{S} \in(0,1)$; that is, the equilibrium is nondegenerate.

An important property of the equilibrium is that the centralized market imposes externality on the decentralized market. Specifically, the marginal valuation $v_{m}$ is positively related to the ask price $a$ as shown in (25). That is, a high ask price discourages buyers from entering the centralized market. Importantly, a higher ask price makes the decentralized market tighter in the sense that the proportion of buyers $\alpha$ is higher as shown in (24). ${ }^{11}$ Moreover, compared to the pure decentralized market economy studied in Section 2, the presence of a centralized market makes the decentralized market tighter as described in the following proposition.

Proposition 5. In any nondegenerate search equilibrium with market makers quoting bid-ask prices $(a, b)$, the decentralized market is tighter than that without intermediaries, in that $\alpha>\gamma$, where $\gamma$ and $\alpha$ are given by (13) and (24), respectively.

The intuition behind this proposition is the following. The introduction of a centralized market lowers the average valuation of buyers in the decentralized market. Therefore, the average total trading surplus is lower and a seller's share is also lower. Since the seller's value must stay equal to $R$, he must meet a buyer more often in the decentralize market (see Eq. (17)). This implies that the proportion $\alpha$ of buyers in the decentralize market must be higher.

Another important property of the equilibrium is described in the following proposition.

Proposition 6. In any nondegenerate search equilibrium with market makers quoting bid-ask prices $(a, b)$, the bid-ask prices and the expected price in the decentralized market satisfy $b<$ $E_{G}[p(v)]<a$. Moreover,

$$
E_{G}[p(v)]=R+\frac{(a-R)(1-\theta)}{2} .
$$

Proposition 6 shows that, as long as the centralized and decentralized market coexist in equilibrium, there exists a positive bid-ask spread. Moreover, the expected price in the decentralized

11 Market tightness is defined as the buyer-seller ratio in the decentralized market. 
market lies between the bid and ask price. The intuition is simple. Any trader faces the following trade-off: He may either wait to transact at a negotiated price in the decentralized market or choose immediate execution at the current bid or ask price in the centralized market. Trading in the decentralized market incurs search and delay costs. Thus, the quoted ask price must include a premium for immediate buying and the bid price must reflect a concession required for immediate sale.

An important condition for the result to hold true is that the two markets coexist in equilibrium. For example, when market makers are competitive, the two markets coexist if the time cost of search and the transaction cost are of comparable magnitude (see Propositions 9-10 below).

Proposition 6 also shows that the average negotiated price in the decentralized market is positively related to the ask price, and hence the bid-ask spread since the bid price is fixed at $R$. To understand this result, observe that there are two opposing effects of the bid-ask spread on the expected negotiated price: An increase in the bid-ask spread discourages some high valuation buyers from entering the centralized market. Hence it raises the average negotiated price. On the other hand, it also raises a seller's payoff since he is able to meet more high valuation buyers. But in the steady state, a seller's payoff is equal to $R$, which does not depend on the bid-ask spread. To maintain this value, the seller must meet a buyer less often. This implies a buyer can meet a seller more often, imposing a positive externality on the buyer's payoff. Thus, he can negotiate a lower price. The proposition shows that the former effect dominates.

In the next two subsections, I will analyze how market makers determine the bid-ask prices and characterize equilibria.

\subsection{Competitive market makers}

Competitive market makers make zero profits. Since a transaction yields profits $(a-b-k)$ and market markers are identical, they all quote the same prices, which are given by $b=R$, and $a=b+k=R+k$. Thus, the bid-ask spread is equal to the transaction cost of market makers.

To solve for the equilibrium with competitive market makers, one only needs to substitute $a=R+k$ into the equilibrium derived in Proposition 4. In particular, the matching probability $\alpha$ is given by

$$
\alpha=\frac{2 R r}{\rho k(1-\theta)} .
$$

The marginal participation type is given by $v_{m}$,

$$
v_{m}=\frac{k(1-\theta)(r+\rho \theta)+\operatorname{Rr}(1-3 \theta)}{(1-\theta) r} .
$$

The following result follows from Proposition 4.

Proposition 7. If the values of parameters $\left(k, r, \rho, \theta, f_{B}, f_{S}\right)$ are such that $a=R+k$ satisfies condition (23), then there is a unique nondegenerate search equilibrium with competitive market makers.

To analyze the equilibrium, I first study some limiting results. 
Proposition 8. For any parameter values $\left(k, r_{0}, \rho, \theta, f_{B}, f_{S}\right)$ satisfying the assumption in Proposition 7 , there exist values $\bar{r}>0$ and $\underline{r}>0$ such that if $r$ converges up (below) to $\bar{r}(\underline{r})$ from $r_{0},{ }^{12}$ then the decentralized (centralized) market is driven out of the economy. If $r$ converges below further to 0 , then the limiting equilibrium becomes Walrasian.

A similar result for $\rho$ can be established and its proof is omitted.

Proposition 9. For any parameter values $\left(k, r, \rho_{0}, \theta, f_{B}, f_{S}\right)$ satisfying the assumption in Proposition 7 , there exist values $\bar{\rho}>0$ and $\rho>0$ such that if $\rho$ converges up (below) to $\bar{\rho}(\underline{\rho})$ from $\rho_{0}$, then the centralized (decentralized) market is driven out of the economy. If $\rho$ converges up further to infinity, then the limiting equilibrium becomes Walrasian.

Propositions 8-9 establish that if either the time cost of search or the contact cost of search is small enough, then all traders prefer to trade in the decentralized market and the centralized market is driven out of the economy. When either type of search cost vanishes, the limiting equilibrium becomes Walrasian. On the other hand, if search cost is large enough, then there is no gain from trading in the decentralized market and all traders prefer to go to the centralized market.

Proposition 10. For any parameter values $\left(k_{0}, r, \rho, \theta, f_{B}, f_{S}\right)$ satisfying the assumption in Proposition 7, there exist values $\underline{k}>0$ and $\bar{k}>0$ such that if $k$ converges below (up) to $\underline{k}(\bar{k})$ from $k_{0}$, then the decentralized (centralized) market is driven out of the economy. If $k$ converges below further to zero, then the limiting equilibrium becomes Walrasian.

Proposition 10 establishes that if the transaction cost of market makers is small enough, or if the bid-ask spread is small enough, then all traders prefer to trade in the centralized market and the decentralized market is driven out of the economy. Moreover, when the bid-ask spread converges to zero, the limiting equilibrium converges to the Walrasian equilibrium. On the other hand, when the bid-ask spread is large enough, no traders go to the centralized market and it is driven out of the economy.

I now study comparative statics with respect to parameters $(k, r, \rho, \theta)$. I will focus particularly on trading volume $f_{B}\left(1-v_{m}\right)$ in the centralized market and the expected price in the decentralized market. ${ }^{13}$ One can interpret trading volume as a measure of liquidity or a measure of the market participation.

Proposition 11. Let the assumption in Proposition 7 hold. Then the expected negotiated price in the decentralized market, $E_{G}[p(v)]$, is increasing with $k$ and decreasing with $\theta$.

Proposition 11 shows that the expected negotiated price in the decentralized market is positively related to the transaction cost in the centralized market. That is, it is negatively related to the efficiency in the centralized market. This result follows directly from Proposition 5 since the competitive bid-ask spread is equal to the transaction cost.

\footnotetext{
12 Note that $\bar{r}$ and $\underline{r}$ may depend on parameter values $\left(f_{B}, f_{S}, k, \theta, \rho\right)$. A similar remark applies for Propositions 9-10.

13 It follows from (22) that the trading volume in the decentralized market is given by the difference between the inflows of traders and the trading volume in the centralized market.
} 
Proposition 11 also shows that similar to Proposition 2, the average negotiated price is negatively related to buyers' bargaining power. However, unlike Proposition 2, under competitive market-making, it does not depend on the contact rate $\rho$ and the discount rate $r$. This is because there are two opposing effects in force. An increase in the contact rate raises a buyer's payoff from trading in the decentralized market, and hence lowers the negotiated price (see (1)). However, it also discourages high valuation buyers from entering the centralized market, leaving more high valuation buyers trading in the decentralized market. This raises the average negotiated price. Proposition 11 implies that these two effects offset each other. A similar analysis applies to $r$.

The following proposition establishes properties of the trading volume in the centralized market.

Proposition 12. Let the assumption in Proposition 7 hold. Then the trading volume in the centralized market, $f_{B}\left(1-v_{m}\right)$, is decreasing with $k, \rho$, and increasing in $r$. It is increasing with $\theta$ if $k \rho(1-\theta)^{2}<2 R r$.

Proposition 12 implies that trading volume in the centralized market is negatively related to the bid-ask spread (or transaction cost $k$ ), and positively related to the search cost reflected by the contact rate $\rho$ and the discount rate $r$ in the decentralized market. This result is intuitive and simply says that trading volume should reflect the relative competitive position of the two markets.

One may expect that if the bargaining power of the buyers $\theta$ increases, then buyers can negotiate low prices as described in Proposition 11. As a result, they should find the decentralized market more attractive and there should be less trade in the centralized market. Surprisingly, Proposition 12 implies that in general there is no monotonic relation between trading volume in the centralized market and the bargaining power of the buyers $\theta$. The intuition is as follows. Ceteris paribus, increasing the buyers' relative bargaining power $\theta$ raises a buyer's payoff and lowers a seller's payoff when they trade in the decentralized market. However, as shown earlier, a seller's steady state payoff must be equal to $R$, which does not depend on the bargaining power. To maintain this payoff, a seller must meet a buyer more often. That is, the proportion of buyers $\alpha$ must be higher as implied by Eq. (17). This imposes a negative externality to the buyers since it implies that the decentralized market becomes tighter. Thus, if this negative effect dominates the preceding positive effect, then the buyer's payoff from trading in the decentralized market actually falls. This leads more buyers to entering the centralized market. This case happens if the decentralized market is relatively less efficient than the centralized market in the sense that $r$ is high or $\rho$ is small, or $k$ is small, as required by the assumption.

\subsection{Monopolistic market maker}

I now analyze the case with a monopolistic market maker. A search equilibrium with a monopolistic market maker is defined as the equilibrium described in Section 3.3 for which the ask price is selected by the market maker so as to maximize profits. Formally, since I focus on steady states, the ask price is determined by the following static problem

$$
\max _{a}(a-b-k) f_{B}\left(1-v_{m}\right),
$$

subject to (23), where $b=R$ and $v_{m}$ is given in (25). 
The monopolistic market maker faces the following trade-off: Increasing the ask price raises the profits from a transaction. But it lowers the number of transactions since some buyers may find the decentralized market is more attractive. Since it follows from (25) that $v_{m}$ is a linear function of $a$, the profit function in (30) is quadratic. The following proposition establishes the existence and uniqueness of search equilibrium and characterizes the equilibrium ask price.

Proposition 13. If the expression given in (31) satisfies condition (23), then there is a unique nondegenerate search equilibrium with a monopolistic market maker. The equilibrium ask price is given by

$$
a=\frac{k}{2}+\frac{r+2 R \theta \rho}{2(r+\theta \rho)}+\frac{\operatorname{Rr}(1+\theta)}{2(r+\theta \rho)(1-\theta)},
$$

which is higher than the competitive ask price $R+k$.

Proposition 13 demonstrates that the bid-ask spread is positively related to the transaction cost $k$ of the market maker. The intuition is similar to that for the case with competitive market makers: A monopolistic market maker makes positive profits, and hence the bid-ask spread must cover the transaction cost. Importantly, (31) also reveals that there exists a positive bid-ask spread even though there is no transaction cost in the centralized market. This result is in line with Proposition 6.

Proposition 13 also implies that the monopolistic market maker sets a wider bid-ask spread than competitive market makers. Consequently, it follows from Propositions 4 and 6 that the average negotiated price in the decentralized market is higher, and trading volume in the centralized market is lower. This result is intuitive. The high ask price set by the monopolistic market maker discourages some high valuation buyers from entering the centralized market and lowers its trading volume. These high valuation buyers raise the average negotiated price in the decentralized market.

Under monopolistic market-making, one can use Propositions 4 and 13 to establish similar limiting results to Propositions $8-10$. The proof is also similar. Hence, I omit a formal statement of the results and the proof. The general intuition should be clear: If one market is sufficiently efficient relative to the other market, it should attract all traders and drives out the other market from the economy. However, unlike competitive market-making, when the transaction cost $k$ goes to zero, the decentralized market does not disappear under monopolistic market-making. This is because there still exists monopoly inefficiency in the centralized market.

The following proposition establishes comparative static properties.

Proposition 14. Let the assumption in Proposition 13 hold. Then:

(i) the bid-ask spread $a-b$, increases with $r$, decreases with $\rho$, and increases with $\theta$ if $2 R\left(r+\theta^{2} \rho\right)>(1-\theta)^{2} \rho(1-R)$

(ii) the expected negotiated price in the decentralized market increases with $k$ and $r$, decreases with $\rho$, and decreases with $\theta$ if $r^{2}+r \rho(1-R)+k(r+\rho \theta)^{2}>3 r^{2} R$; and,

(iii) the trading volume in the centralized market $f_{B}\left(1-v_{m}\right)$ increases with $r$, decreases with $k$ and $\rho$, and increases with $\theta$ if $k \rho(1-\theta)^{2}<2 R r$.

Part (i) of Proposition 14 reveals that the bid-ask spread is positively related to the search frictions in the decentralized market represented by $r$ and $\rho$. This is because when $r$ is decreased 
or $\rho$ is increased (search cost is lower), buyers get higher payoffs from trading in the decentralized market. To attract buyers to trade in the centralized market, the market maker must lower the bid-ask spread.

Importantly, part (i) also shows that there is no general monotonic relation between the bid-ask spread and the bargaining power. The intuition is similar to that described after Proposition 11: An increase in $\theta$ raises a buyer's payoff from trading in the decentralized market, and hence encourages him to trade in that market. On the other hand, more searching buyers make it more difficult for a buyer to find a seller, and thus lowers the buyer's payoff. The overall effect on a buyer's payoff is ambiguous, and hence the effect on a buyer's participation decision is also ambiguous. Consequently, the impact on bid-ask spread is also ambiguous since the market maker adjusts the bid-ask spread so as to attract traders to trade in the centralized market.

Part (ii) of Proposition 14 shows that the expected negotiated price in the decentralized market is positively related to $k$, as in the case of competitive market makers described in Proposition 11. However, unlike Proposition 11, the expected negotiated price is also negatively related to the contact rate $\rho$ and positively related to the discount rate $r$. This is because there are two opposing effects in force as discussed after Proposition 11. Proposition 14 demonstrates the relative efficiency effect dominates. Finally, part (ii) also implies that there is no monotonic relation between the average negotiated price and the buyer's bargaining power. The intuition is similar to that described above.

Finally, part (iii) of Proposition 14 shows that the trading volume in the centralized market is negatively related to $k$ and $\rho$, and positively related to $r$. Moreover, there is no monotonic relationship with $\theta$. This result is similar to that for competitive market makers shown in Proposition 12. The intuition is also similar: Trading volume or liquidity should reflect the relative efficiency of the two markets.

\section{Welfare}

An important question is what is the welfare implication of the market fragmentation. To answer this question, one has to adopt a welfare criterion. Since the Walrasian equilibrium of the benchmark frictionless economy is in the flow sense of Gale (1987), I also adopt the flow sense welfare criterion.

The social welfare $W_{w}$ in the Walrasian equilibrium is given by the total buyer and seller surplus:

$$
W_{w}=f_{B}(1-R) \int_{R}^{1}(v-R) \frac{\mathrm{d} v}{1-R}+f_{S} R=\frac{f_{S}(1+R)}{2} .
$$

This is the first-best value. The social welfare $W_{c}$ in the pure centralized market equilibrium described at the beginning of Section 3 is given by the total buyer and seller surplus plus market maker profits:

$$
\begin{aligned}
W_{c} & =f_{B}(1-a) \int_{a}^{1}(v-a) \frac{\mathrm{d} v}{1-a}+f_{S}(a-b-k)+f_{S} b \\
& =f_{S}\left(\frac{1+R}{2}-k\right),
\end{aligned}
$$


where I use the fact that the market maker sets the ask price $a=R$. Note that this welfare criterion does not depend on the market structure in the centralized market. It is clearly less than the first best value due to the loss of transaction costs.

Consider next the social welfare in the search equilibrium without market makers described in Section 2. It is given by

$$
W_{d}=(1-R) f_{B} \int_{R}^{1} V_{B}(v) \frac{\mathrm{d} v}{1-R}+f_{S} R=f_{S}\left[\int_{R}^{1} V_{B}(v) \frac{\mathrm{d} v}{1-R}+R\right] .
$$

Clearly, $W_{d}<W_{w}$ due to search frictions. The following proposition compares this welfare with that in the pure centralized market.

Proposition 15. If $k<(>) \frac{1}{2} r \frac{3 R \theta-R+1-\theta}{(1-\theta)(r+\rho \theta)}$, then $W_{c}>(<) W_{d}$.

The intuition behind Proposition 15 is as follows. From (33) and (34), one can see that search frictions reduce buyer surplus in the pure decentralized market economy, but transactions costs reduce social welfare in the pure centralized market economy. Thus, which one is bigger depends on the relative efficiency in the two markets described by the parameters $k, \rho$, and $r$.

Turn to the equilibrium where centralized and decentralized markets coexist. In this equilibrium, the social welfare $W_{f}$ is equal to the buyer and seller surplus from trading in the centralized and decentralized markets plus market maker profits:

$$
\begin{aligned}
W_{f}= & \left(v_{m}-R\right) f_{B} \int_{R}^{v_{m}} U_{B}(v) \frac{\mathrm{d} v}{v_{m}-R}+\left(1-v_{m}\right) f_{B} \int_{v_{m}}^{1}(v-a) \frac{\mathrm{d} v}{1-v_{m}} \\
& +f_{S} U_{S}+\left(1-v_{m}\right) f_{B}(a-b-k) \\
= & f_{S}\left[\int_{R}^{v_{m}} U_{B}(v) \frac{\mathrm{d} v}{1-R}+\int_{v_{m}}^{1}(v-R) \frac{\mathrm{d} v}{1-R}+R-\frac{1-v_{m}}{1-R} k\right],
\end{aligned}
$$

where I have used (21)-(22) and the fact that $U_{S}=R=b$ to derive the equality.

It is ready to analyze the question as to whether market fragmentation improves social welfare. The following intuition is natural: The introduction of a centralized market to the decentralized market facilitates immediacy of trade and hence should improve social welfare. On the other hand, the fragmentation of a centralized market should lower social welfare since there are search frictions in the decentralized market. However, I will show that both claims are generally not true.

Consider first the question of whether the opening of a centralized market improves the social welfare in the pure decentralized market economy. Rewrite (34) as

$$
W_{d}=f_{S}\left[\int_{R}^{v_{m}} V_{B}(v) \frac{\mathrm{d} v}{1-R}+\int_{v_{m}}^{1} V_{B}(v) \frac{\mathrm{d} v}{1-R}+R\right] .
$$

It is clear from (35) and the fact $V_{B}(v)<v-R$ for $v \geqslant v_{m}$ that high valuation buyers $v \geqslant v_{m}$ benefit from immediacy of trade. However, the centralized market imposes negative externality on the decentralized market in the sense that it makes the latter market tighter (see Proposition 5). Thus, buyers in the decentralized market are worse off, i.e., $V_{B}(v)>U_{B}(v)$ for 
Table 1

Parameter values

\begin{tabular}{llllll}
\hline$f_{B}$ & $f_{S}$ & $r$ & $k$ & $\rho$ & $\theta$ \\
\hline 1250 & 1000 & 0.05 & 0.00025 & 200 & 0.5
\end{tabular}

$v \in\left[R, v_{m}\right]$. Moreover, market making incurs transaction costs. Consequently, the social welfare in the economy where the two markets coexist may not be higher than that in the pure decentralized market economy. To illustrate this point, I consider some simple numerical examples. Set parameter values as in Table 1. Suppose market makers are competitive and denote by $W_{c m}$ the associated social welfare. For the base case parameter values in Table 1, I find that $W_{d}=599.700<W_{c m}=599.765$. That is, the presence of a competitive centralized market improves social welfare. In particular, the benefit of immediacy net of transaction cost is equal to 0.076 , which exceeds the externality cost 0.011 . However, if I set $\rho=400$, I find $W_{d}=599.850>W_{c m}=599.844$. This is because the benefit of immediacy net of transaction cost $(0.003)$ is less than the externality cost $(0.009)$. Thus, if the decentralized market is relatively efficient enough, then the opening of a competitive centralized market may lower social welfare.

Consider next the question of whether the fragmentation of a centralized market improves social welfare in the pure centralized market economy. Rewrite $W_{c}$ as

$$
W_{c}=f_{S}\left[\int_{R}^{v_{m}}(v-R) \frac{\mathrm{d} v}{1-R}+\int_{v_{m}}^{1}(v-R) \frac{\mathrm{d} v}{1-R}+R-k\right] .
$$

Compared with (35), it is clear that the centralized market provides immediacy of trade and hence improves the welfare of traders with valuation $v \in\left[R, v_{m}\right]$ as $v-R>U_{B}(v)$. However, since in a completely centralized market all traders trade with market makers, the total transaction costs $f_{S} k$ is bigger than that in the two markets economy $f_{S} \lambda_{S} k$. The following proposition shows that if the bid-ask spread after the market fragmentation is small enough, then the second effect dominates. In particular, under competitive market making, the fragmentation of centralized markets always improves social welfare.

Proposition 16. If the bid-ask spread $(a-R)$ in the two markets economy satisfies $(a-R)<(>)$ $2 k$, then $W_{f}>(<) W_{c}$. In particular, under competitive market-making, $W_{c m}>W_{c}$.

I finally address the following questions: What is the constrained socially optimal bid-ask spread? How do the equilibria under competitive and monopolistic market-making compare with the constrained social optimum?

Again the bid price must be equal to $R$ such that sellers are indifferent between trading in the two markets. Thus, the social planner chooses the ask price $a$ only so as to maximize $W_{f}$ given in (35). This is equivalent to choosing the cutoff value $v_{m}$ since it follows from (25) that $v_{m}$ is positively related to $a$.

Proposition 17. The socially optimal cutoff value $v_{m}^{*}$ satisfies

$$
v_{m}^{*}-R-k=U_{B}\left(v_{m}^{*}\right)+\int_{R}^{v_{m}^{*}} \frac{\partial U_{B}(v)}{\partial v_{m}} \mathrm{~d} v .
$$


This value is bigger than the cutoff value under competitive market-making. Furthermore, the socially optimal bid-ask spread is wider than that under competitive market-making.

This proposition implies that under competitive market-making, the bid-ask spread is too narrow and there is too much entry into the centralized market, compared with the constrained social optimum. The main reason is that the social planner internalizes the externality of the bid-ask prices on the decentralized market. This externality effect is captured by the second term on the right-hand side of (38). Specifically, when $v_{m}$ or $a$ changes, it follows from (24) that the matching probability $\alpha$ changes. Thus, it follows from (19) that the buyer value function $U_{B}(v)$ also changes. By contrast, competitive market makers completely ignore this externality effect when setting the ask price $a=R+k$.

I now consider the welfare implication of monopolistic market-making. When the monopolistic market maker chooses the profit maximizing ask price (31), he or she takes into account the impact of the bid-ask prices on the traders' participation decisions. That is, unlike competitive market makers, the monopolistic market maker takes into account the externality effect, even though this externality is not fully internalized. Thus, one should expect that monopolistic market-making may improve social welfare. I now use a simple numerical example to illustrate this point. According to the parameter values in Table 1, the outcome of the constrained social optimum is described in row 2 of Table 2 . In particular, the constrained efficient social welfare is equal to 599.778, the bid-ask spread is equal to 0.00035 , and trading volume in the centralized market is equal to 624.69. Rows 3-4 of Table 2 reveal that under monopolistic market-making, the social welfare is closer to the constrained social optimum. Moreover, the monopolistic market maker appropriates the welfare gain 0.076 at the expense of the traders in the sense that the traders' total surplus 599.695 is less than the value, 599.765, under competitive market-making. Table 2 also reveals that monopolistic market-making implies a too wide bid-ask spread and two little trading volume in the centralized market.

The above analysis has important policy implications. First, there can be welfare gain from increasing the bid-ask spread in a perfectly competitive centralized market. This suggests that taxing transactions in the competitive centralized market might improve social welfare. Another policy that might improve social welfare might be to give some monopoly power to market makers. This might explain why the specialists system on the New York Stock Exchange could be socially useful. ${ }^{14}$

Table 2

Comparison of social welfare

\begin{tabular}{lllll}
\hline & Bid-ask spread & Negotiated price & Volume & Social \\
& $a-b$ & $E_{G}[p(v)]$ & $f_{B}\left(1-v_{m}\right)$ & welfare \\
\hline Social optimum & 0.00035 & 0.20009 & 624.69 & 599.778 \\
Monopolistic MM & 0.00042 & 0.20011 & 449.48 & 599.772 \\
Competitive MM & 0.00025 & 0.20006 & 874.69 & 599.765 \\
\hline
\end{tabular}

Notes. Row 2 lists equilibrium outcome for the constrained social optimum. Row 3 lists equilibrium outcome under competitive market-making. Row 4 lists equilibrium outcome under monopolistic market-making. The parameter values are given in Table 1.

14 In a model with asymmetric information, Glosten (1989) argues that the specialists system on the NYSE may improve social welfare. The intuition is that the monopolistic specialist can set prices that on average maximize profits. Thus, the market breakdown problem as in the market-for-lemons problem does not arise. 
Recently, there is a considerable debate about the consequence of the merger between NYSE and Archipelago. ${ }^{15}$ Some commentators think that this consolidation may reduce competition and liquidity in off-floor trading. The model here suggests that the consolidation may improve social welfare because it may partially internalize the negative externalities.

\section{Conclusion}

This paper provides a search model of centralized and decentralized trade. The model has a number of testable implications. As mentioned in the introduction, some are consistent with empirical and experimental evidence.

The main results of the paper can be summarized as follows. First, a positive bid-ask spread exists if the centralized and decentralized markets coexist in equilibrium. Moreover, the average negotiated price in the decentralized market is inside the bid-ask spread. Second, under monopolistic market-making, the bid-ask spread is positively related to the transaction cost, search frictions, and average negotiated price. Third, liquidity in the centralized market measured by trading volume is negatively related to the bid-ask spread and positively related to search frictions. Fourth, several limiting results and convergence to the Walrasian equilibrium are established.

Finally, perhaps the most important and surprising result is about the welfare implications. Specifically, I show that the fragmentation of a centralized market improves social welfare if the bid-ask spread after the fragmentation is small enough. However, the opening of a centralized market in a decentralized market economy may not improve social welfare. More interestingly, compared to the competitive market-making, monopolistic market-making may improve social welfare, because it partially internalizes the externalities of bid-ask prices on the decentralized market.

The model is highly stylized and may not describe perfectly any specific market in reality. It intends to capture in a simple manner some crucial elements of trades in the centralized and decentralized markets. As a result, the model can be extended in a number of dimensions.

First, since the model is stylized and it is difficult to gather decentralized trade data, an experimental study is helpful for testing the model. Related experimental studies have been carried out by Campbell et al. (1991), Lamoureux and Schnitzlein (1997), and Neeman and Vulkan (2003b). Apparently, further work along this line is interesting.

Second, in the model, the only benefit of the centralized market is its publicity of prices and immediacy of trade. Centralized markets have other important advantages such as economies of scale and network externalities. A simple way to capture these advantages is to assume that the transaction cost $k$ decreases with the volume of trade.

Finally, in order to keep the model tractable and to derive analytical results, I assume that sellers are homogeneous, which seems reasonable when a single homogeneous asset is traded. It would be interesting to consider the case where sellers are heterogeneous. For example, they may have different costs of holding the asset.

\section{Acknowledgments}

I thank Rasmus Lentz and Zvika Neeman for helpful discussions. I also thank two anonymous referees and the editor, Gary Hansen, for useful comments. In particular, an anonymous referee

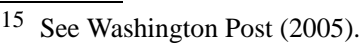


has provided detailed and thoughtful comments and suggestions, and should recognize in the text some of his or her own writing.

\section{Appendix A. Proofs}

Proof of Proposition 1. See the main text.

Proof of Proposition 2. Substituting the expression for $\gamma$ in (13) into (11) yields

$$
p(v)=R+\frac{r(3 \theta R-R+1-\theta)}{(r+\rho \theta)(1-R)}(v-R) .
$$

Simple algebra delivers $\frac{\partial p(v)}{\partial \rho}<0$, and

$$
\begin{aligned}
& \frac{\partial p(v)}{\partial r}=\frac{\theta \rho(3 R \theta-R+1-\theta)}{(r+\rho \theta)^{2}(1-R)}(v-R), \\
& \frac{\partial p(v)}{\partial \theta}=-r \frac{r+\rho-\rho R-3 R r}{(r+\rho \theta)^{2}(1-R)}(v-R) .
\end{aligned}
$$

By assumption (14), one can verify that $\frac{\partial p(v)}{\partial r}>0$ and $\frac{\partial p(v)}{\partial \theta}<0$.

Proof of Proposition 3. Substituting (13) into (11) and letting $r$ converge to 0, or letting $\rho$ converge to infinity, one obtains that the limiting price is given by $R$ for all $v$. Thus, all traders in the market trade at the Walrasian price. Thus, the limiting equilibrium is Walrasian.

Proof of Proposition 4. To derive the equilibrium, observe first that the bid price $b=R$ as discussed in the main text. Next, substituting $U_{S}=R$ and (19) into (15) yields the bargained price between buyer $v$ and a seller given in (26). I now solve for the matching probability $\alpha$ and the cutoff value $v_{m}$. To this end, I turn to the seller's problem (17). Because of the cutoff nature of buyers' choice, the distribution of buyers in the decentralized market $G$ is uniform over $\left[R, v_{m}\right]$ and its density is $\frac{\mathrm{d} v}{v_{m}-R}$. Thus, substituting (26) into (17) yields

$$
r U_{S}=\rho \alpha(1-\theta) \int_{R}^{v_{m}}\left[v-U_{S}-U_{B}(v)\right] \frac{\mathrm{d} v}{v_{m}-R} .
$$

Substituting $U_{S}=R$ and $U_{B}$ given in (19) into the above equation yields

$$
R=\frac{\rho \alpha(1-\theta)}{r+\rho(1-\alpha) \theta} \int_{R}^{v_{m}}[v-R] \frac{\mathrm{d} v}{v_{m}-R} .
$$

Use this equation to solve for $v_{m}$,

$$
v_{m}=R \frac{2(r+\theta \rho(1-\alpha))+\rho \alpha(1-\theta)}{\rho \alpha(1-\theta)} .
$$

On the other hand, use Eq. (20) to derive

$$
v_{m}=\frac{1}{r}[(r+\rho \theta(1-\alpha)) a-\rho \theta(1-\alpha) R] .
$$


Equate the right-hand sides of Eqs. (A.4) and (A.3) to deliver a quadratic equation for $\alpha$. Solving this equation yields two roots. One root is given by $\frac{r+\rho \theta}{\rho \theta}$, which must be ruled out since it is bigger than 1. The other root is given by (24). Substituting this root into (A.3) yields (25). Finally, once $v_{m}$ and $\alpha$ are obtained, one can use (21)-(22) to derive $\left(N_{B}, N_{S}, \lambda_{S}\right)$, and an equilibrium is constructed.

Proof of Proposition 5. In a nondegenerate search equilibrium with market makers quoting bidask prices $(a, b)$, the cutoff value satisfies $v_{m} \in(R, 1)$. From (12) and (A.2), it is straightforward to verify $\alpha>\gamma$.

Proof of Proposition 6. Since a buyer with valuation $v$ in the support of $G,\left[R, v_{m}\right]$, prefers to buy the asset at price $p(v)$, at some random time $\tau_{b}$, rather than to buy the asset immediately at the price $a$ in the centralized market,

$$
v-a \leqslant E\left[\mathrm{e}^{-r \tau_{b}}\right](v-p(v)) .
$$

Since $E\left[\mathrm{e}^{-r \tau_{b}}\right]<1$, it follows that $p(v)<a$ for all $v \in\left[R, v_{m}\right]$. Taking expectation with respect to $G$ yields $a>E_{G}[p(v)]$. Similarly, one can show that $E_{G}[p(v)]>b .{ }^{16}$ Finally, substituting (24) into (26) and taking expectation, one can easily derive (27).

Proof of Proposition 7. See the main text.

Proof of Proposition 8. Taking other parameter values as given, view $v_{m}$ as a function of $r$. By assumption there is a value $r_{0}>0$ such that $v_{m}\left(r_{0}\right) \in(R, 1)$. One can also show that $v_{m}(r) \rightarrow \infty$ as $r \rightarrow 0$. Thus, there is a positive solution to the quadratic equation $v_{m}(r)=1$. Take $\underline{r}$ as the maximum solution. When $r$ converges to $\underline{r}, v_{m}$ converges to 1 and hence no buyers go to the centralized market. The model then reduces to that in Section 2. By Proposition 2, the economy becomes Walrasian if $r$ converges below to 0 .

Now consider increasing $r$ from $r_{0}$. By (28) and (29), when $r$ is sufficiently large, either $\alpha$ will exceed 1 or $v_{m}$ will decrease below $R$. For both cases, all buyers prefer to go to the centralized market and the decentralized market disappears.

Proof of Proposition 9. The proof is similar to that of Proposition 8, and is omitted.

Proof of Proposition 10. By (28) and (29), when $k$ is sufficiently small, either $\alpha$ tends to 1 , or $v_{m}$ tends to $R$. Thus, all buyers enter the centralized market and there is no trade in the decentralized market. When $k$ converges below further to zero, then there is no bid-ask spread; that is, $a=b$. Moreover, only buyers with valuation $v \geqslant a$ enter the centralized market. Thus, to maintain a steady state, the following flow condition must be satisfied

$$
f_{B}(1-a)=N_{B}=N_{S}=f_{S} .
$$

This implies that $a=R$ and the limiting equilibrium becomes Walrasian.

Finally, it follows from (29) that $v_{m}$ increases with $k$. Thus, there exists a value $\bar{k}>0$ such that $v_{m}$ converges to 1 when $k$ tends to $\bar{k}$. This implies that if $k$ is large enough, then no traders go to the centralized market, and hence the centralized market disappears.

$\overline{16}$ I thank an anonymous referee for suggesting this simpler proof. 
Proof of Proposition 11. Since $a=R+k$, it follows from Proposition 6 that

$$
E_{G}[p(v)]=R+\frac{k(1-\theta)}{2} .
$$

Thus, $E_{G}[p(v)]$ is increasing with $k$ and decreasing with $\theta$.

Proof of Proposition 12. It follows from straightforward differentiation of $v_{m}$ given in (29).

Proof of Proposition 13. Substituting (25) into (30) yields a quadratic function of $a$. The firstorder condition gives the maximizer given in (31). I denote it by $a_{m}$. If it satisfies condition (23), then by Proposition 4 there is a unique search equilibrium with a monopolistic market maker quoting the ask price given in (31). To show that this ask price is bigger than $R+k$, observe that there are two roots, denoted by $a_{1}$ and $a_{2}$, for the profit function (30). The maximizer $a_{m}$ is between these two roots. Note that one root $a_{1}$ is such that $v_{m}=1$, and the other root $a_{2}=$ $R+k$. The maximizer $a_{m}$ must satisfies $a_{2}<a_{m}<a_{1}$. Otherwise, suppose $a_{1}<a_{m}<a_{2}$. Since (25) implies that $v_{m}$ increases with $a$, the value of $v_{m}$ at $a_{m}$ must exceed 1 . This leads to a contradiction.

Proof of Proposition 14. Let $a$ be the expression given in (31). For part (i), simple algebra implies that

$$
\begin{aligned}
\frac{\partial a}{\partial r} & =\frac{(3 R \theta-\theta-R+1) \theta \rho}{2(r+\theta \rho)^{2}(1-\theta)}, \\
\frac{\partial a}{\partial \rho} & =-\frac{(3 R \theta-\theta-R+1) r \theta}{2(r+\theta \rho)^{2}(1-\theta)}, \\
\frac{\partial a}{\partial \theta} & =\frac{r\left(2 R\left(r+\theta^{2} \rho\right)-(1-\theta)^{2} \rho(1-R)\right)}{2(r+\theta \rho)^{2}(1-\theta)^{2}} .
\end{aligned}
$$

By assumption,

$$
\frac{r}{r+\rho \theta}+\frac{R \theta(2 r+\rho(1-\theta))}{(1-\theta)(r+\rho \theta)}-a>0 \text {. }
$$

Thus,

$$
\begin{aligned}
& \frac{r}{r+\rho \theta}+\frac{R \theta(2 r+\rho(1-\theta))}{(1-\theta)(r+\rho \theta)}-\frac{r+2 R \theta \rho}{2(r+\theta \rho)}-\frac{R r(1+\theta)}{2(r+\theta \rho)(1-\theta)} \\
& =\frac{1}{2}(r+\theta \rho)^{-1}(1-\theta)^{-1}(3 R \theta-\theta-R+1) r>0 .
\end{aligned}
$$

The desired result then follows.

Consider part (ii). By (27), the expected negotiated price $E_{G}[v(p)]$ increases with the ask price $a$. The desired comparative statics result for $k, r$, and $\rho$ follows from part (i). Finally, the comparative statics result for $\theta$ follows from the assumption and the following equation,

$$
\frac{\partial(a-R)(1-\theta)}{\partial \theta}=-\frac{r^{2}(1-3 R)+r \rho(1-R)+k(r+\rho \theta)^{2}}{2(r+\rho \theta)^{2}} .
$$

Finally, consider part (iii). Substituting the expression given in (31) into (25) yields an expression for $v_{m}$. Differentiating this expression yields: 


$$
\begin{aligned}
\frac{\partial v_{m}}{\partial r} & =-r^{-2} \rho \theta k<0, & \frac{\partial v_{m}}{\partial \rho} & =\frac{k}{2 r} \theta>0, \\
\frac{\partial v_{m}}{\partial k} & =\frac{1}{2 r}(r+\theta \rho)>0, & \frac{\partial v_{m}}{\partial \theta} & =\frac{k \rho(1-\theta)^{2}-2 R r}{r(1-\theta)^{2}} .
\end{aligned}
$$

The desired result then follows from the assumption.

Proof of Proposition 15. Substituting (10) and (13) into (33)-(34), and simplifying yield

$$
\begin{aligned}
W_{c}-W_{d} & =f_{S}\left(\frac{1-R}{2}+R-k\right)-f_{S}\left[\int_{R}^{1} V_{B}(v) \frac{\mathrm{d} v}{1-R}+R\right] \\
& =\frac{1}{2} r \frac{3 R \theta-R+1-\theta}{(1-\theta)(r+\rho \theta)}-k .
\end{aligned}
$$

The desired result follows from the assumption.

Proof of Proposition 16. Use (35) and (33) to derive

$$
\begin{aligned}
W_{f}-W_{c}= & f_{S}\left[\int_{R}^{v_{m}} U_{B}(v) \frac{\mathrm{d} v}{1-R}+\int_{v_{m}}^{1}(v-R) \frac{\mathrm{d} v}{1-R}+R-\frac{1-v_{m}}{1-R} k\right] \\
& -f_{S}\left[\int_{R}^{v_{m}}(v-R) \frac{\mathrm{d} v}{1-R}+\int_{v_{m}}^{1}(v-R) \frac{\mathrm{d} v}{1-R}+R-k\right] \\
= & f_{S} k \frac{v_{m}-R}{1-R}-f_{S} \int_{R}^{v_{m}}\left(v-R-U_{B}(v)\right) \frac{\mathrm{d} v}{1-R} \\
= & \frac{v_{m}-R}{1-R} f_{S}\left(k-\frac{r}{r+\theta \rho(1-\alpha)} \frac{v_{m}-R}{2}\right) \\
= & \frac{v_{m}-R}{1-R} f_{S}[k-(a-R) / 2],
\end{aligned}
$$

where the third equality follows from substitution of (19), and the last equality follows from substitution of (24) and (25). Since for competitive market makers $a-R=k$, the desired result follows from the assumption.

Proof of Proposition 17. Taking first-order condition for (35) yields (38). Using (19), (24), and (25), one can easily show that $\frac{\partial U_{B}(v)}{\partial v_{m}}>0$. Let the constrained social optimal ask price be $a^{*}$. By (20), $v_{m}^{*}-a^{*}=U_{B}\left(v_{m}^{*}\right)$. Thus, one can rewrite (38) as

$$
a^{*}-R-k=\int_{R}^{v_{m}^{*}} \frac{\partial U_{B}(v)}{\partial v_{m}} \mathrm{~d} v>0 .
$$

It follows that $a^{*}>R+k$, the ask price under competitive market-making. Moreover, since the cutoff value $v_{m}$ increases with $a$ by (25), one obtains the desired comparison result for the cutoff value. 


\section{References}

Amihud, Y., Mendelson, H., 1980. Dealership markets: Market making with inventory. Journal of Financial Economics 8, 31-53.

Bagehot, W., 1971. The only game in town. The Financial Analysts Journal 27, 12-14.

Biais, B., 1993. Price formation and equilibrium liquidity in fragmented and centralized markets. Journal of Finance 48, $157-184$

Campbell, J., LaMaster, S., Smith, V.L., Boening, M.V., 1991. Off-floor trading, disintegration, and the bid-ask spread in experimental markets. Journal of Business 64, 495-522.

Demsetz, H., 1968. The cost of transacting. Quarterly Journal of Economics 82, 33-53.

Diamond, P.A., 1982. Wage determination and efficiency in search equilibrium. Review of Economic Studies 49, 217227.

Duffie, D., Garleanu, N., Pedersen, L.H., 2003a. Valuation in over-the-counter asset markets. Working paper. GSB, Stanford University.

Duffie, D., Garleanu, N., Pedersen, L.H., 2003b. Over-the-counter marketmaking. Working paper. Stanford University.

Gale, D., 1987. Limit theorems for markets with sequential bargaining. Journal of Economic Theory 43, $20-53$.

Garman, M., 1976. Market microstructure. Journal of Financial Economics 3, 257-275.

Gehrig, T., 1993. Intermediation in search markets. Journal of Economics and Management Strategy 2, 97-120.

Glosten, L.R., 1989. Inside trading, liquidity, and the role of the monopolist specialist. Journal of Business 62, 211-236.

Glosten, L.R., Milgrom, P.R., 1985. Bid, ask, and transaction prices in a specialist market with heterogeneously informed traders. Journal of Financial Economics 14, 71-100.

Harris, L., 2003. Trading and Exchanges: Market Microstructure for Practitioners. Oxford Univ. Press, New York.

Ho, T., Stoll, H.R., 1981. Optimal dealer pricing under transactions and return uncertainty. Journal of Financial Economics 9, 47-73.

Hosios, A.J., 1990. On the efficiency of matching and related models of search and unemployment. Review of Economic Studies 57, 279-298.

Kyle, K., 1985. Continuous auctions and insider trading. Econometrica 53, 1315-1335.

Lamoureux, C.G., Schnitzlein, C.R., 1997. When it's not the only game in town: The effect of bilateral search on the quality of a dealer market. Journal of Finance 52, 683-712.

Li, Y., 1998. Middlemen and private information. Journal of Monetary Economics 42, 131-159.

Lippman, S.A., McCall, J.J., 1986. An operation measure of liquidity. American Economic Review 1, 43-55.

Madhavan, A., 1995. Consolidation, fragmentation, and the disclosure of trading information. Review of Financial Studies 8, 579-604.

Masters, A., 2004. Efficiency of intermediation in search equilibrium. Working paper. SUNY, Albany.

Mendelson, H., 1987. Consolidation, fragmentation, and market performance. Journal of Financial and Quantitative Analysis 22, 189-207.

Mortensen, D.T., 1982. Property rights and efficiency in mating, racing, and related games. American Economic Review 72, 968-979.

Mortensen, D.T., Wright, R., 2002. Competitive pricing and efficiency in search equilibrium. International Economic Review 43, 1-20.

Neeman, Z., Vulkan, N., 2003a. Markets versus negotiations: The predominance of centralized markets. Working paper. Boston University.

Neeman, Z., Vulkan, N., 2003b. Markets versus negotiations: An experimental analysis. Working paper. Boston University.

Pagano, M., 1989. Trading volume and asset liquidity. Quarterly Journal of Economics 104, 255-274.

Rubinstein, A., Wolinsky, A., 1985. Equilibrium in a market with sequential bargaining. Econometrica 53, 1133-1150.

Rubinstein, A., Wolinsky, A., 1987. Middlemen. Quarterly Journal of Economics 102, 581-593.

Rust, J., Hall, G., 2003. Middlemen versus market makers: A theory of competitive exchange. Journal of Political Economy $111,353-403$.

Shevchenko, A., 2004. Middlemen. International Economic Review 45, 2004.

Spulber, D.F., 1996. Market making by price-setting firms. Review of Economic Studies 63, 559-580.

Stoll, H.R., 2000. Friction. Journal of Finance 55, 1479-1514.

Vayanos, D., Wang, T., Search anf endogenous concentration of liquidity in asset markets. Working paper. MIT.

Washington Post, 2005. NYSE-archipelago deal may affect trading. The Associated Press, April 25.

Weill, P., 2004. Leaning against the wind. Working paper. NYU. 